\title{
Experimental Demyelination and Axonal Loss Are Reduced in MicroRNA-146a Deficient Mice
}

OPEN ACCESS

Edited by:

Maja Jagodic,

Karolinska Institute (KI),

Sweden

Reviewed by:

Rishein Gupta,

University of Texas at

San Antonio, United States

Nicolas Fissolo,

Vall d'Hebron Research

Institute, Spain

${ }^{*}$ Correspondence:

Zsolt llles

zsolt.illes@rsyd.dk

Specialty section:

This article was submitted to

Multiple Sclerosis and

Neuroimmunology,

a section of the journal

Frontiers in Immunology

Received: 22 October 2017 Accepted: 26 February 2018

Published: 12 March 2018

Citation:

Martin NA, Molnar V, Szilagyi GT, Elkjaer ML, Nawrocki A, Okarmus J,

Wlodarczyk A, Thygesen EK,

Palkovits M, Gallyas F Jr., Larsen MR,

Lassmann H, Benedikz E, Owens T,

Svenningsen AF and Illes Z (2018)

Experimental Demyelination

and Axonal Loss Are Reduced

in MicroRNA-146a Deficient Mice.

Front. Immunol. 9:490

doi: 10.3389/fimmu.2018.00490
Nellie A. Martin', Viktor Molnar', Gabor T. Szilagyi³, Maria L. Elkjaer', Arkadiusz Nawrocki", Justyna Okarmus', Agnieszka Wlodarczyk ${ }^{5}$, Eva K. Thygesen ${ }^{1}$, Miklos Palkovits ${ }^{6}$, Ferenc Gallyas Jr. ${ }^{3,7,8}$, Martin R. Larsen ${ }^{4}$, Hans Lassmann ${ }^{9}$, Eirikur Benedikz', Trevor Owens ${ }^{5}$, Asa F. Svenningsen ${ }^{5}$ and Zsolt Illes ${ }^{1,5,10 *}$

${ }^{1}$ Department of Neurology, Odense University Hospital, Odense, Denmark, ${ }^{2}$ Department of Genetics, Cell- and Immunobiology, Semmelweis University, Budapest, Hungary, ${ }^{3}$ Department of Biochemistry and Clinical Chemistry, University of Pécs, Pécs, Hungary, ${ }^{4}$ Department of Biochemistry and Molecular Biology, University of Southern Denmark, Odense, Denmark, ${ }^{5}$ Department of Neurobiology Research, Institute for Molecular Medicine, University of Southern Denmark, Odense, Denmark, ${ }^{6}$ Laboratory of Neuromorphology and Human Brain Tissue Bank, Microdissection Laboratory, Semme/weis University, Budapest, Hungary, ${ }^{7}$ Szentagothai Research Centre, University of Pécs, Pécs, Hungary, ${ }^{8}$ NuclearMitochondrial Interactions Research Group, Hungarian Academy of Sciences, Budapest, Hungary, ${ }^{9}$ Center for Brain Research, Medical University of Vienna, Vienna, Austria, ${ }^{10}$ Department of Clinical Research, University of Southern Denmark, Odense, Denmark

Background: The cuprizone (CPZ) model of multiple sclerosis (MS) was used to identify microRNAs (miRNAs) related to in vivo de- and remyelination. We further investigated the role of miR-146a in miR-146a-deficient (KO) mice: this miRNA is differentially expressed in MS lesions and promotes differentiation of oligodendrocyte precursor cells (OPCs) during remyelination, but its role has not been examined during demyelination.

Methods: MicroRNAs were examined by Agilent Mouse miRNA Microarray in the corpus callosum during CPZ-induced demyelination and remyelination. Demyelination, axonal loss, changes in number of oligodendrocytes, OPCs, and macrophages/microglia was compared by histology/immunohistochemistry between KO and WT mice. Differential expression of target genes and proteins of miR-146a was analyzed in the transcriptome $(4 \times 44 \mathrm{~K}$ Agilent Whole Mouse Genome Microarray) and proteome (liquid chromatography tandem mass spectrometry) of CPZ-induced de- and remyelination in WT mice. Levels of proinflammatory molecules in the corpus callosum were compared in WT versus KO mice by Meso Scale Discovery multiplex protein analysis.

Results: miR-146a was increasingly upregulated during CPZ-induced de- and remyelination. The absence of miR-146a in $\mathrm{KO}$ mice protected against demyelination, axonal loss, body weight loss, and atrophy of thymus and spleen. The number of $\mathrm{CNP}^{+}$oligodendrocytes was increased during demyelination in the miR-146a KO mice, while there was a trend of increased number of NG2+ OPCs in the WT mice. miR-146a target genes, SNAP25 and SMAD4, were downregulated in the proteome of demyelinating corpus callosum in WT mice. Higher levels of SNAP25 were measured by ELISA in the corpus callosum of miR-146a KO mice, but there was no difference between $\mathrm{KO}$ and WT mice 
during demyelination. Multiplex protein analysis of the corpus callosum lysate revealed upregulated TNF-RI, TNF-RII, and CCL2 in the WT mice in contrast to KO mice. The number of $\mathrm{Mac}^{+}$and $\mathrm{Iba1}^{+}$macrophages/microglia was reduced in the demyelinating corpus callosum of the $\mathrm{KO}$ mice.

Conclusion: During demyelination, absence of miR-146a reduced inflammatory responses, demyelination, axonal loss, the number of infiltrating macrophages, and increased the number of myelinating oligodendrocytes. The number of OPCs was slightly higher in the WT mice during remyelination, indicating a complex role of miR-146a during in vivo de- and remyelination.

Keywords: cuprizone, miR-146a, miR-181b, miR-193a, demyelination, remyelination, multiple sclerosis

\section{INTRODUCTION}

Multiple sclerosis (MS) is an inflammatory demyelinating disease of the central nervous system [CNS; (1)]. Immunomodulatory treatments are available for prevention of relapses in the relapsing-remitting form of the disease, but treatment options to prevent demyelination are limited (2).

It has been estimated that more than $60 \%$ of all protein-coding mammalian genes can be regulated by microRNAs (miRNA) (3). The functional level of miRNAs can be manipulated in vivo (4), and clinical trials are already running with the intention of either restoring miRNA function by administration of miRNA mimics (5) or inhibiting their function by antimiR oligonucleotids (6). The posttranscriptional regulatory system of microRNAs has been found to be extensively involved in almost all biological processes, including those essential in the pathology of MS (7). Many microRNAs are differentially regulated in response to MS in brain lesions $(8,9)$, whole blood $(10,11)$, isolated blood cells $(12,13)$, plasma and serum $(14,15)$, and cerebral spinal fluid $(16$, 17). Still, the actual role of these differentially expressed miRNAs in MS pathology has been not extensively explored. One of the miRNAs that is differentially regulated in different tissues and cells in MS is miR-146a: it has been found to be upregulated in brain lesions (8), serum (18) and blood-derived immune cells $(13,19)$. The role of miR-146a as a negative regulator of immune activation is well established $(20,21)$. miR-146a is involved in a negative feedback loop: it is induced by NF-kB, but also inhibits the activation of NF-KB (22). miR-146a is involved in cell death and survival; in glioma cells: overexpression of miR-146a suppressed cell survival, proliferation, and migration, whereas inhibition resulted in improved migration potential $(23,24)$. miR-146a amplified the effect of a G-actin-sequestering peptide to promote OPC differentiation in vitro, and its overexpression in neural progenitor cells increased their differentiation into OPCs $(25,26)$. Recently, infusion of miR-146a mimics into demyelinated corpus callosum after 5-week administration of cuprizone (CPZ) promoted differentiation of OPCs into myelinating oligodendrocytes (27).

Oral administration of the copper chelator bis-cyclohexanoneoxalyldihydrazone (CPZ) leads to demyelination most pronounced in the corpus callosum, whereas discontinuation of $\mathrm{CPZ}$ results in rapid remyelination (28). The mechanism of CPZ-induced demyelination is not fully understood but mitochondrial dysfunction and oxidative stress to which oligodendrocytes are particularly sensitive is suspected $(29,30)$. The blood-brain barrier remains intact, and the absence of $\mathrm{T}$ and $\mathrm{B}$ cells do not affect CPZ-induced demyelination (31). Therefore, de- and remyelination can be examined as relatively separated processes unmasked from the contribution of adaptive immune responses, in contrast to another commonly used MS model, experimental autoimmune encephalomyelitis (EAE) $(28,30)$.

Considering the differential expression of microRNAs in different tissues and compartments of patients with MS, here we used the CPZ mouse model of MS to identify and investigate miRNAs involved in de- and remyelination. By using microarray, we identified three miRNAs differentially expressed during experimental de- and remyelination that were also reported to be differentially expressed in MS lesions (8). One of these miRNAs, miR-146a can have both pro- anti-apoptotic effect in different cells, including OPCs $(26,32)$, and had a unique expression profile during de- and remyelination in our experiments. This miRNA has been recently investigated in the CPZ model, and its injection promoted differentiation of OPCs into myelinating oligodendrocytes during remyelination in wild-type (WT) mice (27). Here, we investigated, if the absence of miR-146a influences demyelination in the CPZ model by using miR-146a-deficient (KO) mice.

\section{MATERIALS AND METHODS}

\section{miR-146a KO Mice}

miR-146a knockout (KO) mice were purchased from the Jackson Laboratory (ME, USA). This mouse strain was generated on a C57BL/6 background in Dr. David Baltimore's laboratory, California Institute of Technology (33). Mice were bred at the Biomedical Laboratory, SDU according to protocols and guidelines approved by the Danish Animal Health Care Committee (2014-15-00369). All animal experiments complied with the EU Directive 2010/63/EU for animal experiments. Female KO mice aged 7-8 weeks were included in the experiments. At this age, miR-146a KO mice do not display a visible autoimmune or inflammatory phenotype (33). 


\section{Cuprizone-Induced Demyelination and Remyelination}

Cuprizone (Sigma Aldrich, MO, USA) was delivered orally: powdered standard chow was mixed with $0.2-0.4 \% \mathrm{CPZ}$. To induce demyelination, CPZ was administrated to 7- to 8-week-old mice for 4 weeks ( 4 weeks demyelination: $4 \mathrm{wd}$ ). Remyelination was examined at two time-points: acute remyelination induced by 4 weeks CPZ feeding followed by 2 days of regular diet (2 days remyelination: $2 \mathrm{dr}$ ), and full remyelination induced by 4 weeks CPZ feeding followed by 2 weeks of regular diet (2 weeks remyelination: $2 \mathrm{wr}$ ). Control mice were kept on a normal diet. During experiments, mice were weighed every second day to control that mice lost no more than maximum $20 \%$ of their body weight. Experiments were terminated by euthanizing mice with an overdose of pentobarbital (Glostrup Apotek, Glostrup, Denmark) followed by perfusion with $4 \%$ paraformaldehyde (PFA) for staining applications, or phosphate buffered saline for all other experiments.

\section{Extraction of Whole RNA and Quantitative PCR (qPCR)}

For the removal of the corpus callosum, the brains were removed from the skull, immediately frozen, and cut into coronal serial sections (section thickness $200 \mu \mathrm{m}$ ). By using a stereomicroscope, the corpus callosum was cut out of the sections with a fine Graefe-knife, along its rostro-caudal extension. The samples were collected in ice-cold Eppendorf-tubes and stored frozen until used. RNA was extracted by the miRNeasy micro Kit (Qiagen, Valencia, CA, USA). The quantity and quality of total RNA was assessed by NanoDrop ND-1000 spectrophotometer (NanoDrop Technologies, Wilmington, DE, USA) and Agilent 2100 Bioanalyzer (Agilent Technologies, Palo Alto, CA, USA), respectively. Only those samples were used for microarray experiments that gave $>8.0$ for RNA integrity number, showed a clear gel image, and no DNA contamination was observed on the histogram.

To measure miRNA expression, primer sets for specific miRNA assays and sno135 endogenous control and the MicroRNA Reverse Transcription Kit (Life Technologies, Thermo Scientific, CA, USA) were utilized following the manufacturer's protocol. Briefly, $10 \mathrm{ng}$ of each total RNA sample were transcribed by MultiScribe Reverse Transcriptase. qPCR was carried out using Applied Biosystems 7000 Real-Time PCR System. Specific TaqMan chemistry primers for reverse transcription and qPCR were acquired from Life Technologies (Thermo Scientific) (miR146a ID: 000468, snoRNA135 ID: 001230, CA, USA). Reverse transcription was conducted under the following conditions: $30 \mathrm{~min}$ at $16^{\circ} \mathrm{C}, 30 \mathrm{~min}$ at $42^{\circ} \mathrm{C}, 5 \mathrm{~min}$ at $85^{\circ} \mathrm{C}$, cool down to $4^{\circ} \mathrm{C}$; and for $\mathrm{qPCR}$ analyses the following conditions were applied: $2 \mathrm{~min}$ at $50^{\circ} \mathrm{C}, 10 \mathrm{~min}$ at $95^{\circ} \mathrm{C}, 40$ cycles of $15 \mathrm{~s}$ at $95^{\circ} \mathrm{C}$ followed by $1 \mathrm{~min}$ at $60^{\circ} \mathrm{C}$ according the manufacturer's instructions. The relative expression of each miRNA was calculated from the equation 2- $\Delta \mathrm{Ct}$, where $\Delta \mathrm{Ct}=$ mean $\mathrm{Ct}($ miRNA) - mean Ct(internal control) (where Ct is the threshold cycle for a sample). All reagents and instruments for qPCR were purchased from Applied Biosystems Inc. except when otherwise indicated.

\section{miRNA Microarray}

For microRNA profiling, the Agilent Mouse miRNA Microarray Kit (G4472A, $8 \times 15 \mathrm{k}$ ) was applied according to the manufacturer's instruction (version 1.0) with 100-100 ng quality-checked total RNA. The labeled samples were hybridized for $20 \mathrm{~h}$ at $55^{\circ} \mathrm{C}$. The arrays were scanned with an Agilent DNA Microarray Scanner BA, the signal quantification was carried out by Feature Extraction 10.7 Image Analysis Software and data were further analyzed by Genespring GX10.0. The microarray data are deposited in NCBI Gene Expression Omnibus with accession GSE100662.

\section{Meso Scale Discovery Multiplex Electrochemiluminescent Assay}

Cytokine levels in the corpus callosum were measured by the Meso Scale Discovery (MSD, USA) electrochemiluminescence proinflammatory mouse V-Plex Plus Kit (IL-1 $\beta$, IL-4, IL-6, IL-10, TNF), a MULTI-SPOT 4 spot cytokine costume plate (MIP1 $\alpha$, VEGF, and MMP9) and a MULTI-SPOT 2 spot cytokine costume plate (TNF-RI and TNF-RII). We used a SECTOR Imager 6000 (Meso Scale Discovery) Plate Reader, and data were analyzed using the MSD Discovery Workbench software according to the manufacturer's instructions. Results are presented relative to the total protein concentration of the individual samples.

\section{ELISA}

SMAD4 and SNAP25 protein levels in the corpus callosum were examined and compared between miR-146a KO mice and WT by ready-made Sandwich ELISA kits according to the manufacturer's instructions (Nordic Biosite, OKEH03425 and EKM1284, respectively). A Molecular Devices, Vmax kinetic microplate reader was used to analyze the results, and results are presented relative to the total protein concentration of the individual samples.

\section{Histopathology}

Brains were postfixed in 4\% PFA overnight before they were embedded in paraffin. Then, $8 \mu \mathrm{m}$ coronal sections were obtained at the levels of 161, 181, 209, and 221 (34). Demyelination was evaluated using Luxol fast blue staining with cresyl violet. Axonal pathology was examined by Bielschowsky staining. The sections in the corpus callosum were overlaid by a 100-point grid at a magnification of $20 \times$ in the microscope and the number of points, each representing $2.5 \mathrm{~mm}^{2}$, located within the lesion was counted. First, the size of the entire lesion was determined (total lesion). Next, the size of the area, which showed remyelination (evenly thin myelin sheaths) was measured. Immunocytochemistry was performed on paraffin sections as described before (35) without antigen retrieval using antibodies against Ibal (WAKO \#01919741), Mac3 (Becton \& Dickinson \#553322), NG2 (Millipore AB 5320), and CNP (Sternberger Monoclonals SMI 91). Stained cells were counted in sections overlaid with a morphometric grid in the ocular lens. The cell count values represent cells $/ \mathrm{mm}^{2}$.

\section{Proteomics}

The proteomic analysis was performed in pentaplicate. The corpus callosum was dissected from fresh-frozen brain obtained 
from mice during demyelination, acute remyelination (2 days), full remyelination (2 weeks), and controls (five mice in each group). The samples were treated with protease and phosphatase inhibitors, ultracentrifuged to remove cell debris and nanostructures and subsequently the total protein content was estimated by amino acid composition analysis. The proteins in the supernatant were reduced with DTT, alkylated with iodoacetamide and digested with trypsin as described elsewhere (36). The purified peptides from each pool were labeled with one of the iTRAQ reagent according to the manufactures protocol and respective labeled samples were mixed in 1:1 proportion (based on the amino acids composition analysis). In order to enrich for phosphopeptides and glycosylated peptides, the TiSH protocol was applied (37). Peptide samples were fractioned and analyzed by LC-MS/MS using a nano-Easy LC (Thermo Fisher Scientific) coupled with either a Q-Exactive or Velos mass spectrometers (Thermo Fisher Scientific, Bremen, Germany). All peptides fractions were resuspended in $0.1 \% \mathrm{FA}$ and loaded onto a $2-\mathrm{cm}$ $100 \mu \mathrm{m}$ inner diameter pre-column using the nano-Easy LC. Peptides were eluted directly onto the analytical column using a gradient of $0-34 \%$ buffer B (90\% Acetonitrile, $0.1 \%$ FA) over 30-120 min depending on the UV intensity of the individual HILIC fractions. All LC-MS/MS runs were performed using an analytical column of $20 \mathrm{~cm} \times 75 \mu \mathrm{m}$ inner diameter fused silica, packed with C18 material (Dr. Maisch, Ammerbuch-Entringen, Germany). The MS settings for Q-Exactive instrument were as follows: full MS: resolution at 60,000, AGC target 1e6, Maximum IT $100 \mathrm{~ms}$, and for data-dependent MS/MS of the top 12 most intense ions: resolution at 15,000, AGC target 2e4, Maximum IT $100 \mathrm{~ms}$, isolation window $1.2 \mathrm{~m} / z$, fixed first mass $110.0 \mathrm{~m} / z$, NCE: 30, Intensity threshold 1e4. For the analysis on Velos instrument, the settings were similar, but top 7 most intense ions were selected for MS/MS fragmentation at 7,500 resolution, NCE 35. The MS raw files were processed and search in Mascot and SEQUEST through the Proteome Discoverer 2.1 software (Thermo Fisher Scientific). Database searches were performed using the following parameters: Precursor mass tolerance of 10 parts per million (ppm); MSMS mass tolerance of $0.05 \mathrm{Da}$; Enzyme: trypsin and up to two missed cleavages were allowed.

\section{Experimental Design and Statistical Analysis}

If not otherwise stated, statistical tests were performed using Prism 7 software (GraphPath, USA, CA, USA) and quantitative data are presented as mean \pm SEM. Exact $p$-values are specified for all ANOVA tests when $p>0.0001$, and $p<0.05$ is considered significant. Each ANOVA test is followed by an appropriate post hoc test.

Raw miRNA expression data were obtained from 3 to 4 mice pr. group. The microarray data were normalized to the 75th percentile signal intensity and entities showing present call in all samples of a condition were filtered out. Differentially expressed genes were selected when passing the signal intensity filter (entities where at least $100 \%$ of samples in any one out of four conditions have values within cutoff) and showing at least twofold statistically significant change (ANOVA and
Tukey HSD post hoc test, with Benjamini-Hochberg multiple testing correction $p$-value $<0.05$ ) between any of the groups. Validation of miRNA expression by qPCR included five to eight mice in each group and data were analyzed by one-way ANOVA followed by LSD post hoc tests. Body weight, thymus and spleen weight, and lesion size analysis included $4-16,4-8$, and 4-7 mice at each time-point, respectively, and data were analyzed using two-way ANOVA followed by Bonferroni post hoc tests.

For the analysis of the proteome, five mice were included. The ratio $(r)$ for each protein, in any of the three comparisons (any condition to the control) was compared to its SE, such that if $r \leq 1 /(1+2 \mathrm{SE})$ or if $r \geq(1+2 \mathrm{SE})$, the protein has changed. If the protein could be measured only in one or two of the five mice, they were not encountered in this analysis.

ELISA analyses of SMAD4 and SNAP25 and Meso Scale Discovery multiplex analysis of cytokines, chemokines, and TNF receptors included four to eight mice in each group, and data were analyzed using two-way ANOVA followed by Bonferroni post hoc tests.

\section{RESULTS}

\section{Differential Expression of MicroRNAs in the Corpus Callosum During CPZ-Induced Demyelination and Remyelination}

In order to identify microRNAs involved in the pathology of de- and remyelination, we isolated the corpus callosum from mice exposed to $\mathrm{CPZ}$ and conducted an Agilent microarray analysis for 627 miRNAs (data are deposited in NCBI Gene Expression Omnibus with accession GSE100662). We identified three miRNAs, miR-146a, miR-181b, and miR-193a, which were differentially expressed compared to controls confirmed by qPCR (Figure 1). The expression of miR-146a increased in response to $\mathrm{CPZ}$ exposure, and continued to increase during the remyelination phase $(p<0.001$, one-way ANOVA, LSD post hoc test) (Figure 1A). By contrast, the expression level of miR-193a and miR-181b decreased in response to CPZ-induced demyelination and had returned to baseline in the full remyelination phase ( $p<0.001$ and $p<0.01$, respectively, one-way ANOVA, LSD post hoc test) (Figures 1B,C).

Observing (i) a continuous increase in the expression level of miR-146a in contrast to the two other miRNAs, (ii) considering its biological function, and (iii) also the differential expression in brain lesions, body fluids, and cells obtained from MS patients $(8,13,18,19,25-27)$, we further investigated the role of miR$146 \mathrm{a}$ in CPZ-induced de- and remyelination.

\section{Expression of miR-146a in Response to $\mathrm{CPZ}$ in Different Organs}

In addition to analyzing the expression level of miR-146a in the corpus callosum, we also analyzed the expression level in thymus, liver, spleen, and muscle tissue. In contrast to the corpus callosum, we did not see a CPZ-induced increase of miR-146a in any of these organs. The highest expression level of miR-146a among the examined organs was found in the spleen (Figure 2A). 
A

A $\quad$ miR-146a

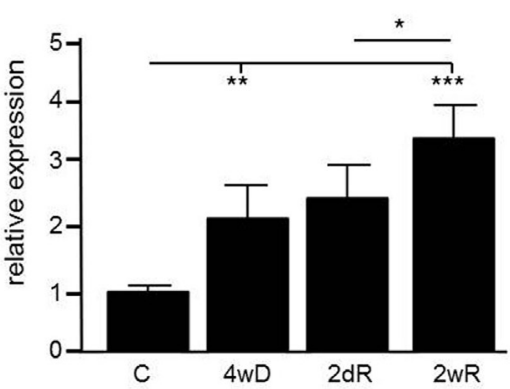

B

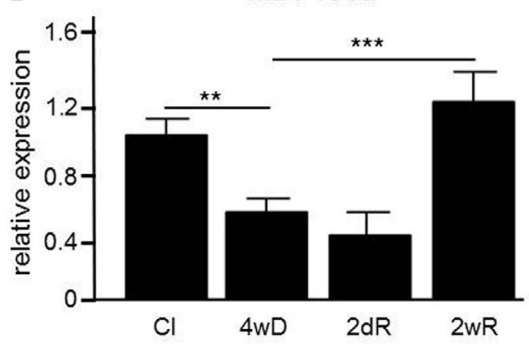

C

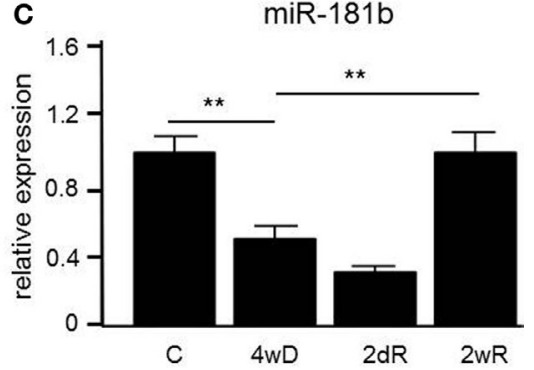

FIGURE 1 | Differential expression of microRNAs (miRNAs) in the corpus callosum of mice during experimental demyelination and remyelination. Using microarray and validation by quantitative PCR (qPCR), three miRNAs, miR-146a (A), miR-193a (B) and miR-181b (C) were differentially regulated in response to CPZ exposure in the corpus callosum. ${ }^{\star} p<0.05,{ }^{* \star} p<0.01,{ }^{\star \star \star} p<0.001, n=5-8$ in each group, one-way ANOVA, mean \pm SEM. Abbreviation: C: un-manipulated controls covering ages of mice used in the de- and remyelinating experiments, 4wD: 4 weeks demyelination. 2dR: 2 days (acute) remyelination, 2 wR: 2 weeks (full) remyelination.
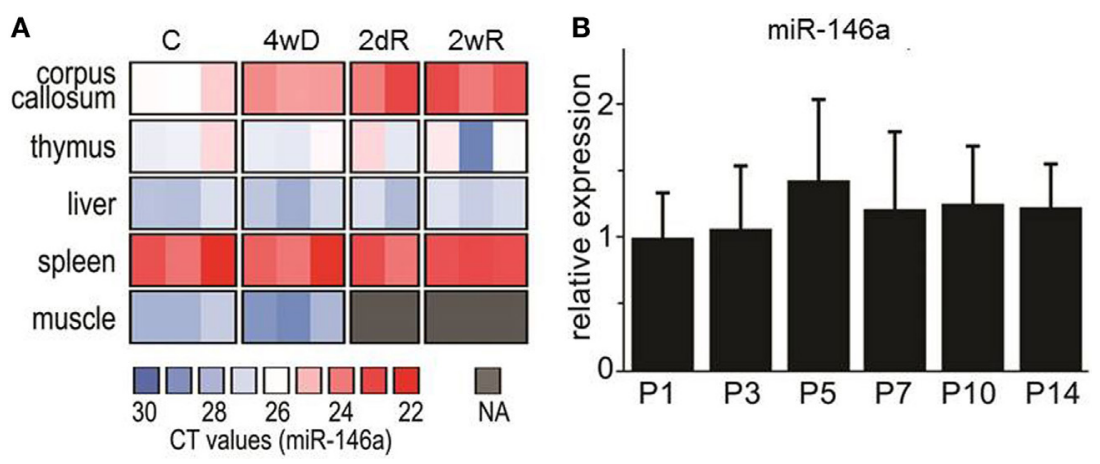

FIGURE 2 | Expression of miR-146 in different organs during cuprizone (CPZ) treatment and during physiological postnatal myelination. (A) The relative expression of miR-146a was determined by qPCR in different organs of individual animals. Abbreviation: C: un-manipulated controls, 4 wD: 4 weeks demyelination. $2 \mathrm{dR}$ : 2 days (acute) remyelination, 2wR: 2 weeks (full) remyelination. (B) The expression of miR-146a in the corpus callosum during physiological myelination (on postnatal days $1-14, \mathrm{P} 1-\mathrm{P} 14)$. The relative expression of miR-146a was determined by qPCR and the results are presented relative to sno135 $(n=4$ in each group, one-way ANOVA, mean \pm SEM).

\section{Expression of miR-146a in the Brain During Physiological Myelination in Postnatal Mice}

In order to investigate, if the level of miR-146a is also increased during physiological myelination, we examined its expression in the corpus callosum isolated from postnatal mice aged $1-14$ days (P1-P14); this is the most critical period for physiological myelination in mice (38). We found no change in the expression of miR-146a, which indicates that the observed increase in response to $\mathrm{CPZ}$ exposure is associated with demyelination pathology (Figure 2B).

To further examine the effect of miR-146 on de- and remyelination in vivo, we used a miR-146a-deficient $(\mathrm{KO})$ strain compared to WT mice in additional experiments (33). 


\section{Systemic Effects of CPZ Exposure in miR-146a-Deficient Mice}

Weight loss is a characteristic systemic effect of $\mathrm{CPZ}$ exposure in mice (29). As expected, both WT and miR-146a KO mice lost weight in response to $\mathrm{CPZ}$ exposure, but miR-146a $\mathrm{KO}$ mice lost significantly less weight than WT mice during the period of demyelination (Figure 3A).

Recently, we observed thymus atrophy as an additional systemic effect of CPZ exposure (39). We, therefore, examined the effect of miR-146a deficiency on thymus weight in response to $\mathrm{CPZ}$ exposure. The spleen weight was also examined, since the highest expression level of miR-146a outside the CNS was found in this organ (Figure 2A). We observed atrophy of the thymus and spleen in the WT mice, whereas atrophy of both organs was less severe or absent in the miR-146a KO mice $(p<0.0001$, spleen, and thymus, respectively) (Figures 3B,C). To determine, if this observation was an artifact of the more pronounced weight loss observed in the WT mice, we also analyzed the organ weight as a percentage of bodyweight, but these analyses showed similar results ( $p<0.00001$, spleen; $p=0.001$, thymus, data not shown). These data indicate that miR-146a KO mice are protected against systemic effects of $\mathrm{CPZ}$ exposure, and this becomes evident after 2 weeks of treatment.

\section{Effect of miR-146a Deficiency on CPZ- Induced Demyelination and Axonal Loss}

Next, we quantified demyelination in the brain of miR-146a $\mathrm{KO}$ and WT mice exposed to CPZ. In the miR-146a KO mice, demyelination and axonal damage was significantly reduced ( $p=0.0001$, two-way ANOVA, Bonferroni post hoc test) (Figures 4A,B).

The number of $\mathrm{CNP}^{+}$myelinating oligodendrocytes was higher in miR-146a KO mice compared to WT mice during demyelination $(p=0.01)$ (Figure 5), while the number of $\mathrm{NG}^{+}$oligodendrocyte precursors was not different. In addition, we found a decreased number of $\mathrm{Mac}^{+}(p<0.05)$ (Figure 5) and a tendency of fewer Iba $1^{+}$cells in the corpus callosum in the $\mathrm{KO}$ mice during demyelination ( $43 \%$ less, $p=0.06$ ). Two weeks after suspending $\mathrm{CPZ}$, the demyelinated lesions were to a large extent remyelinated, contained Iba $1^{+}$cells, but only a minority of these cells was $\mathrm{Mac}^{+}$. There was a modest increase of $\mathrm{CNP}^{+}$cells and decrease of $\mathrm{NG}_{2}{ }^{+}$during remyelination; no difference have been observed between $\mathrm{KO}$ and $\mathrm{WT}$ mice in $\mathrm{CNP}^{+}$oligodendrocytes at this time-point, but we observed a trend of reduced number of $\mathrm{NG}^{+}$ oligodendrocyte precursor cells $(p=0.06)$ (Figure 5).

\section{Expression of Experimentally Validated miR-146a Target Genes and Protein Products in Response to CPZ Exposure}

We extracted a list of experimentally validated miR-146a target genes from the database miRTarBase (40), and compared this list of genes with our transcriptome and proteome datasets during CPZ-induced de- and remyelination. We identified 13 upregulated and 4 downregulated miR-146a target genes among 1,239 differentially expressed genes in the corpus callosum in response to $\mathrm{CPZ}$ exposure determined by a $4 \times 44 \mathrm{~K}$ Agilent Whole Mouse
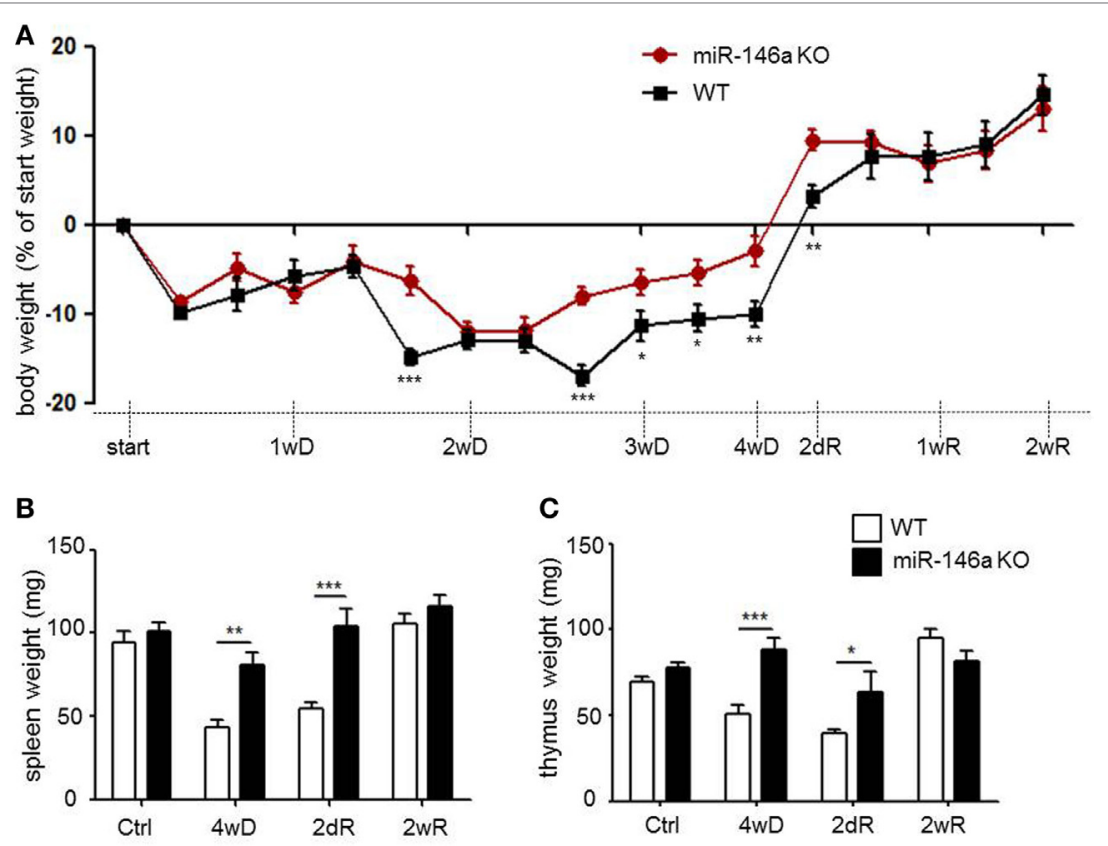

FIGURE 3 Systemic effects of cuprizone in miR-146a-deficient mice. (A) Body weight in response to CPZ exposure in miR-146a KO and WT mice. * $p<0.05$, ${ }^{* *} p<0.01,{ }^{* \star *} p<0.001, n=16-4$ in each group at each time-point (30 mice altogether), two-way ANOVA, mean \pm SEM. (B) Weight of thymus and (C) weight of spleen in response to CPZ exposure in miR-146a KO and WT mice. ${ }^{\star} p<0.05,{ }^{\star \star} p<0.01,{ }^{\star \star \star} p<0.001, n=4-8$ in each group, mean \pm SEM. Abbreviations: 1wD: 1 week demyelination, 2wD: 2 weeks demyelination, 3wD: 3 weeks demyelination, 4wD: 4 weeks demyelination. $2 \mathrm{dR}: 2$ days remyelination, 1 wR: 1 week remyelination, $2 w R: 2$ weeks remyelination. 


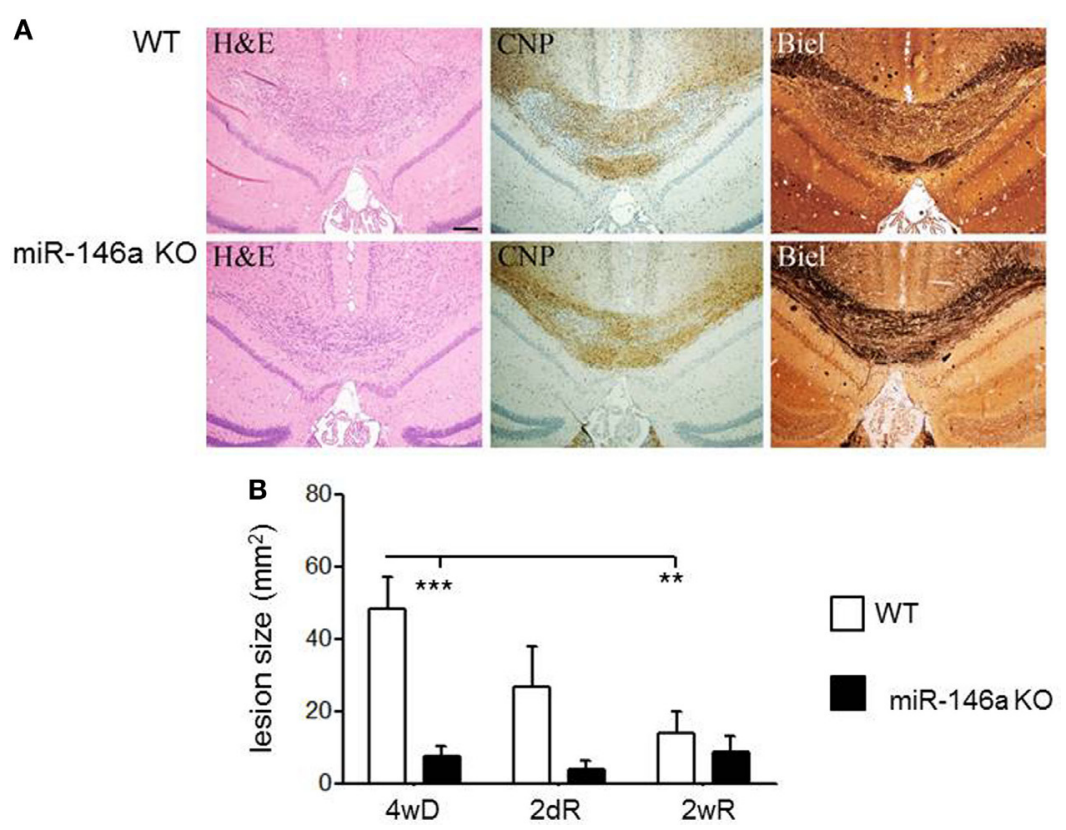

FIGURE 4 | Cuprizone (CPZ)-induced demyelination and remyelination in miR-146-deficient mice. (A) Demyelination and axonal loss after 4 weeks of CPZ exposure in miR-146a KO and WT mice determined by Luxol fast blue (LFB) and Bielschowsky staining (Biel). (B) Quantification of demyelination after 4 weeks CPZ treatment $(4 \mathrm{wD})$ and remyelination after 2 days $(2 \mathrm{dR})$ and 2 weeks remyelination $(2 \mathrm{wR})$ in the corpus callosum of miR-146a KO and WT mice $\left({ }^{\star \star} p<0.01\right.$, ${ }^{\star \star \star} p<0.001$, $n=4-7$ in each group, two-way ANOVA, mean \pm SEM).

A

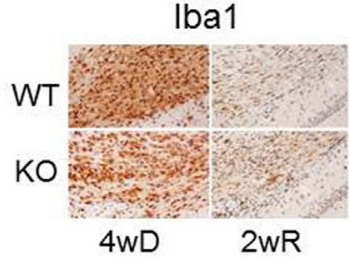

Mac3

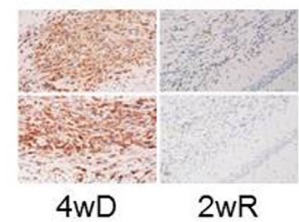

CNP

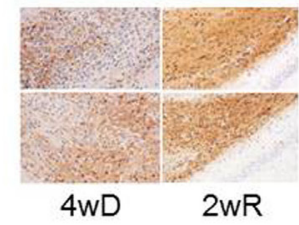

NG2

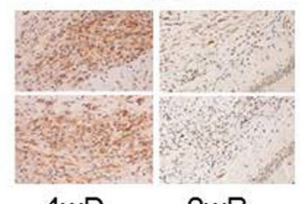

$4 w D \quad 2 w R$
B

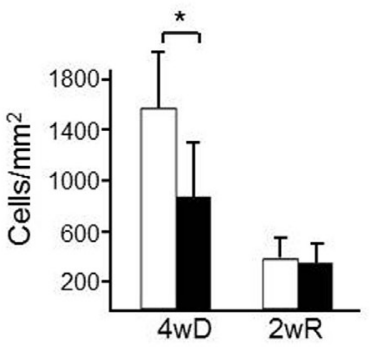

$\mathrm{Mac}^{+}$cells

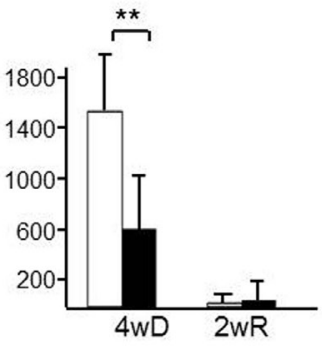

WT
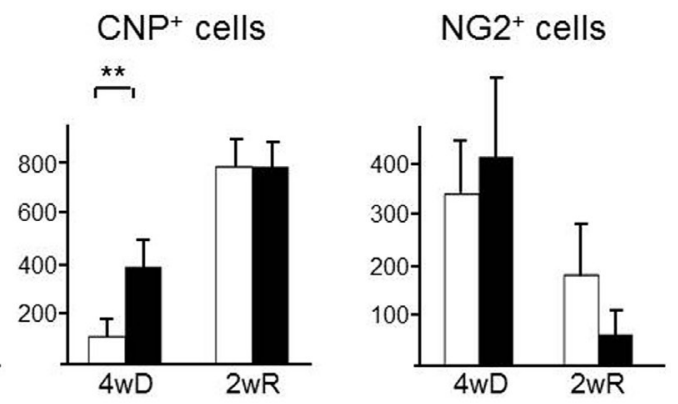

FIGURE 5 | Cellular infiltration in the corpus callosum in miR-146a-deficient mice during cuprizone (CPZ)-induced demyelination and remyelination. (A) Myelinproducing oligodendrocytes (CNP), oligodendrocyte precursor cells (OPCs; NG2), microglia (Iba1), and macrophages (Mac3) in the corpus callosum of WT and miR-146a KO mice exposed to CPZ for 4 weeks demyelination (4wD) or exposed to CPZ for 4 weeks followed by CPZ suspension for 2 weeks remyelination (2wR).

(B) Quantification of $\mathrm{Mac3}^{+}$and $\mathrm{lba1}{ }^{+}$macrophages/microglia, $\mathrm{CNP}^{+}$myelinating oligodendrocytes and NG2+ OPCs in the demyelinating (4 weeks on CPZ, 4wD) and remyelinating (2 weeks on CPZ, 2 wR) corpus callosum of WT and miR-146a KO mice. ${ }^{*} p<0.05$, ${ }^{* *} p<0.01$.

Genome Microarray (unpublished, NCBI Gene Expression Omnibus with accession GSE100663) (Figure 6A). Based on a PANTHER GO enrichment analysis (41) of the differentially regulated miR-146a target genes, inflammatory pathways were highly enriched (Table 1). We also identified 2 downregulated protein products of miR-146a target genes in our proteome dataset 


\begin{tabular}{|c|c|c|c|}
\hline \multicolumn{4}{|l|}{ A } \\
\hline Gene & $4 w D$ & $2 \mathrm{dR}$ & $2 w R$ \\
\hline Spp1 & 83,92 & 14,1 & 25,7 \\
\hline Ccl5 & 6,16 & 12,56 & 23,98 \\
\hline Oasl2 & 6,53 & 9,31 & 9,23 \\
\hline Bcl2a1 & 10,08 & 7,77 & 6,95 \\
\hline Tlr2 & 8,12 & 6,76 & 7,78 \\
\hline Ifit3 & 5,68 & 7,55 & 7,44 \\
\hline Isg15 & 5,48 & 6,46 & 5,75 \\
\hline Cdkn1a & 1 & 10,51 & 1 \\
\hline Rsad2 & 1 & 4,45 & 5,33 \\
\hline Stat1 & 1 & 2,97 & 4,2 \\
\hline Icam1 & 1 & 3,09 & 1 \\
\hline Itgb2 & 1 & 2,02 & 1 \\
\hline Pmaip1 & 1 & 1,88 & 1 \\
\hline Camk2a & 1 & 1 & 0,28 \\
\hline Tmprss5 & 0,26 & 1 & 1 \\
\hline Rhobtb3 & 0,46 & 0,41 & 1 \\
\hline Pacs2 & 0,31 & 0,26 & 1 \\
\hline $\begin{array}{l}\text { B } \\
\text { Protein }\end{array}$ & $4 w D$ & $2 \mathrm{dR}$ & $2 w R$ \\
\hline SMAD4 & 0,65 & 0,28 & 0,86 \\
\hline SNAP25 & 1,26 & 1 & 0,45 \\
\hline
\end{tabular}
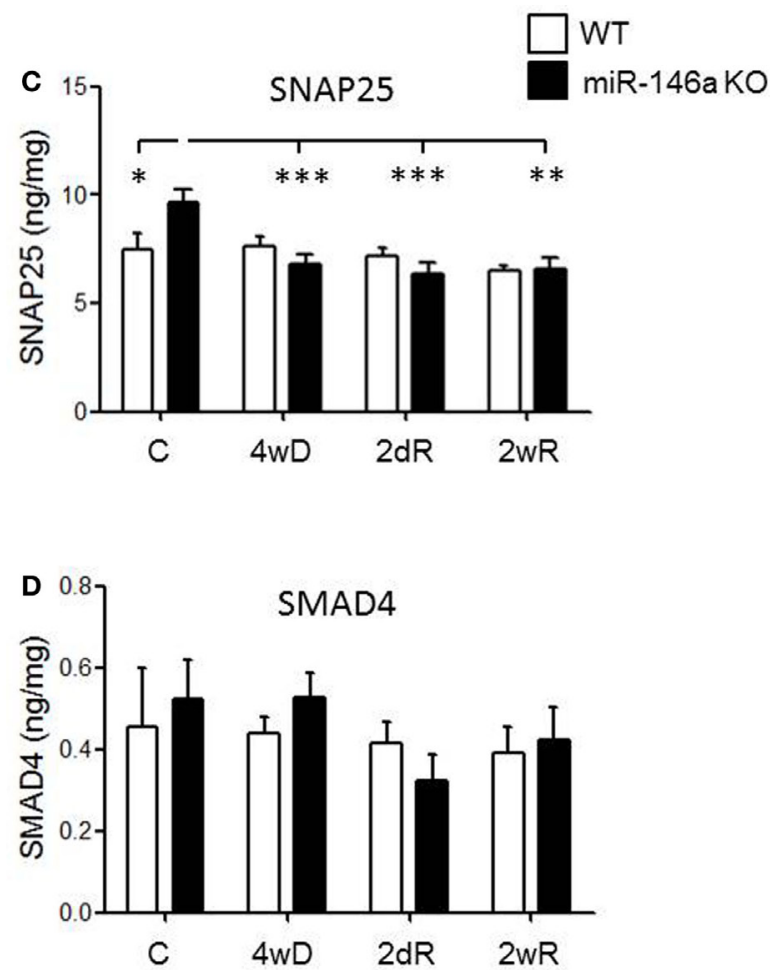

FIGURE 6 | Expression of experimentally validated miR-146a target genes and proteins in response to CPZ exposure in wild-type and miR-146-deficient mice. (A) Expression of miR-146a target genes (miRTarBase) among 1,239 differentially expressed genes in the corpus callosum in response to CPZ exposure determined by a $4 \times 44 \mathrm{~K}$ Agilent Whole Mouse Genome Microarray (NCBI Gene Expression Omnibus with accession GSE100663). (B) The protein expression of validated miR-146a target genes SMAD4 and SNAP25 were downregulated in the corpus callosum of miR-146a KO mice compared to WT mice by liquid chromatography mass spectrometry in response to CPZ. (C) SNAP25 and (D) SMAD4 in corpus callosum lysates of miR-146a KO and WT mice in response to CPZ examined by ELISA. ${ }^{*} p<0.05,{ }^{* *} p<0.01,{ }^{* \star *} p<0.001, n=8-4$ in each group, mean \pm SEM. Abbreviations: C: un-manipulated controls, 4 wD: 4 weeks demyelination. $2 \mathrm{dR}$ : 2 days (acute) remyelination, 2wR: 2 weeks (full) remyelination.

TABLE 1 | Overrepresented biological processes of experimentally validated miR-146a target genes differentially expressed in WT mice during cuprizoneinduced demyelination.

\begin{tabular}{lcc}
\hline GO biological process & Fold enrichment & p-Value \\
\hline Negative regulation of viral genome replication & 93.01 & 0.037 \\
Leukocyte cell-cell adhesion & 90.74 & 0.04 \\
Positive regulation of nitric oxygen & 84.55 & 0.049 \\
biosynthetic process & & \\
Positive regulation of NF-kappaB & 45.09 & 0.014 \\
transcription factor activity & & \\
Defense response to virus & 36.47 & 0.032 \\
Innate immune response & 14.47 & 0.019 \\
Intracellular signal transduction & 7.85 & 0.022 \\
Regulation of apoptotic process & 7.28 & 0.039 \\
\hline
\end{tabular}

Pathway analysis was performed using PANTHER online software. The fold enrichment is the number of genes in our dataset representing the given biological process relative to the number of genes expected by change in a random list of genes.

GO, gene ontology.

(403 differentially regulated proteins, unpublished) (Figure 6B): SMAD4 (mothers against decapentaplegic homolog 4), which is known to be involved in OPC migration and differentiation (42, 43), and SNAP25 (synaptosomal-associated protein 25), which is important in the signal transduction of neurons and neurotransmitter release (44). We used ELISA assays to examine if SMAD4 and SNAP25 were differentially expressed among miR146a KO mice and WT mice in the corpus callosum in response to $\mathrm{CPZ}$ exposure. As expected, we observed an increased protein level of SNAP25 in the corpus callosum of KO mice compared to WT mice in the control group. In the $\mathrm{KO}$ mice, the protein level of SNAP25 was reduced during de- and remyelination, while there was no change in the WT mice $(p<0.01$, two-way ANOVA, Bonferroni post hoc test) (Figure 6C). The SMAD4 protein level was not significantly changed in any of the two strains of mice, and SMAD4 protein was not differentially regulated at any of the examined time-points (Figure 6D).

\section{Expression of Cytokines, Chemokines, and TNF Receptors in Response to CPZ Exposure}

Based on the role of miR-146a in the regulation of inflammatory responses, and the observation that inflammatory pathways were highly enriched among the miR-146a target genes differentially regulated in response to $\mathrm{CPZ}$ exposure, we analyzed and compared the expression level of TNF receptor 1 (TNF-RI) and TNF receptor 2 (TNF-RII) in addition to several inflammatory 
cytokines and chemokines (CCL2, CCL3, CXCL1, IFN-gamma, IL-1beta, IL-2, IL-4, IL-5, IL-6, IL-10, IL-12p70, MMP-9, TNF, and VEGF) in KO versus WT mice.

Both TNF-RI and TNF-RII were significantly upregulated in the WT mice in response to $\mathrm{CPZ}$ exposure, but not in the miR-146a KO mice ( $p<0.001$, respectively, two-way ANOVA, Bonferroni post hoc test) (Figures 7A,B). In fact, during demyelination, the expression level of TNF-RII was significantly lower in the miR-146a KO mice compared to the WT mice $(p<0.01)$ (Figure 7A).

TNF expression was significantly lower during remyelination compared to demyelination in the WT mice $(p<0.01)$. In miR146a KO mice, the highest expression of TNF was found in the control group, which was significantly higher than the expression during acute remyelination $(p<0.05)$. There was no significant difference in TNF expression level at any time-point between the two groups of mice (Figure 7C).

We also observed a significant elevation in the protein levels of chemokine CCL2 in response to CPZ exposure in the WT mice, but not in the miR-146a KO mice $(p<0.001$, two-way ANOVA, Bonferroni post hoc test). In addition, the level of CCL2 was significantly lower in miR-146a KO compared to the WT mice during demyelination $(p<0.01)$ (Figure 7D).

For the additional cytokines and chemokines, we found that IL- $1 \beta$ was upregulated and IL-2, IL-5, IL-6, and IL-12p70 were downregulated in miR-146a KO mice, whereas IL-10 and VEGF were downregulated in both miR-146a KO mice and WT mice in response to $\mathrm{CPZ}$ exposure (Table 2). However, there was no significant difference in expression levels of any of these cytokines and chemokines between miR-146a KO mice and WT mice at the examined time-points (demyelination, acute, and full remyelination).

\section{DISCUSSION}

Here, we used the CPZ mouse model of experimental de- and remyelination to mimic de- and remyelination pathology of MS (30), and examine differentially expressed miRNAs during deand remyelination. Based on a microarray analysis followed by verification with qPCR, we identified three miRNAs, miR-146a, miR-181b, and miR-193a that were differentially expressed in response to $\mathrm{CPZ}$ exposure. All three miRNAs have previously been found to be differentially regulated in MS lesions: miR146a and miR-193a were upregulated in active MS lesions, whereas miR-181b was found to be down regulated in inactive MS lesions (8). So far, four studies investigated expression and effect of miRNAs in the CPZ model, but all used different experimental setups. One study investigated the expression of miR-124 in hippocampal demyelination using dietary CPZ combined with intraperitoneal injection of rapamycin (9).
A

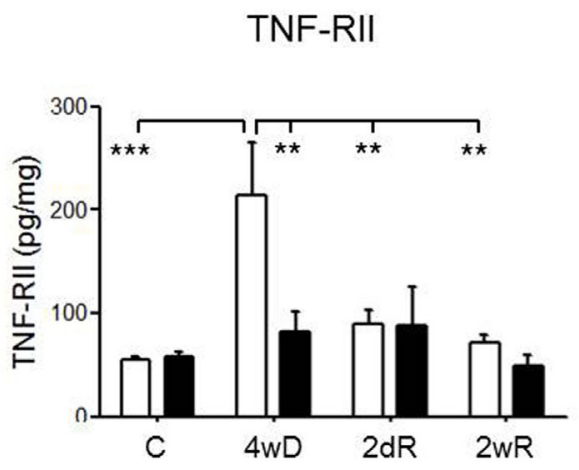

B

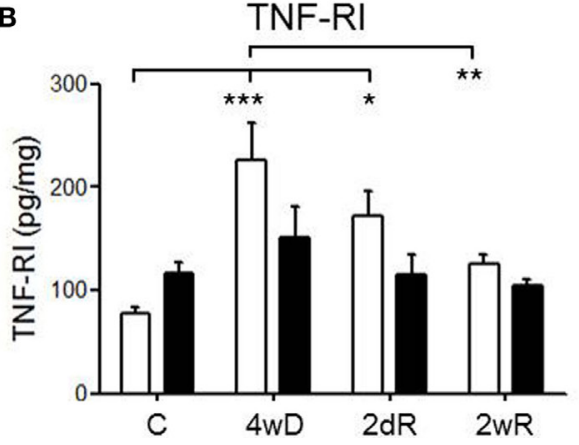

C

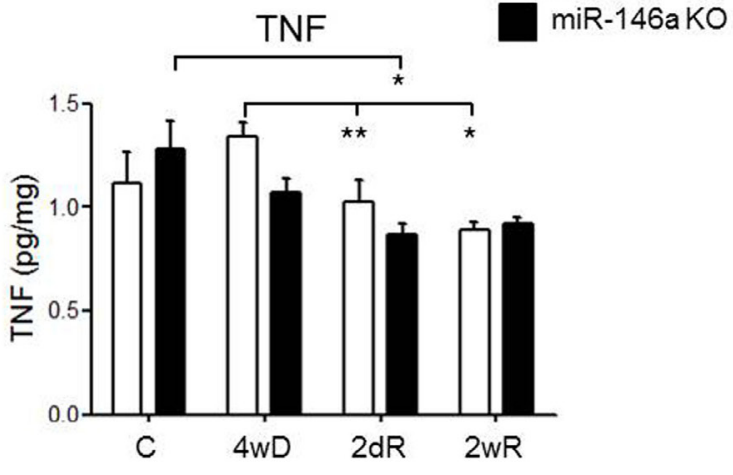

D

$\mathrm{CCL} 2$

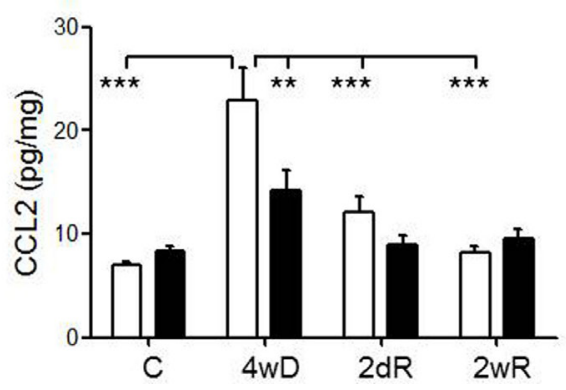

FIGURE 7 | Expression of cytokines, cytokine receptors, and chemokines in the corpus callosum during experimental demyelination and remyelination in miR-146-deficient mice. Expression levels of TNF-RII (A), TNF-RI (B), TNF (C), and CCL2 (D) measured by MSD-electrochemiluminescent assay in the corpus callosum of WT and miR-146a KO mice exposed to CPZ. ${ }^{\star} p<0.05,{ }^{\star \star} p<0.01,{ }^{\star \star \star} p<0.001, n=8-4$ in each group, mean \pm SEM. Abbreviations: C, un-manipulated controls, 4wD, 4 weeks demyelination. $2 \mathrm{dR}, 2$ days (acute) remyelination, $2 \mathrm{wR}, 2$ weeks (full) remyelination. 
TABLE 2 | Expression of cytokines and chemokines in the corpus callosum during cuprizone-induced de- and remyelination in wild-type (WT) and miR-146a KO mice.

\begin{tabular}{|c|c|c|c|c|c|c|c|}
\hline & $4 w d$ & $2 d r$ & $2 w r$ & & $4 w d$ & $2 d r$ & $2 w r$ \\
\hline IL-1beta & & & & IL-12p70 & & & \\
\hline WT & 1.76 & 2.03 & 1.06 & WT & 0.89 & 0.76 & 0.71 \\
\hline $\begin{array}{l}\text { miR- } \\
146 a k O\end{array}$ & $1.92^{\star}$ & 1.21 & 0.91 & $\begin{array}{l}\mathrm{miR}-146 \mathrm{a} \\
\mathrm{KO}\end{array}$ & 0.75 & $0.59^{\star}$ & 0.70 \\
\hline IL-2 & & & & CCL3 & & & \\
\hline WT & 0.85 & 0.78 & 0.76 & WT & 1.3 & 1.24 & 0.78 \\
\hline $\begin{array}{l}\mathrm{miR}-146 \mathrm{a} \\
\mathrm{KO}\end{array}$ & $0.71^{*}$ & $0.60^{\star \star}$ & $0.62^{*}$ & $\begin{array}{l}\mathrm{miR}-146 \mathrm{a} \\
\mathrm{KO}\end{array}$ & 0.82 & 0.65 & 0.64 \\
\hline IL-5 & & & & CXCL1 & & & \\
\hline WT & 0.83 & 0.78 & 0.74 & WT & 1.29 & 1.01 & 0.86 \\
\hline $\begin{array}{l}\mathrm{miR}-146 \mathrm{a} \\
\mathrm{KO}\end{array}$ & $0.69^{\star \star}$ & $0.58^{\star \star \star}$ & $0.60^{\star \star}$ & $\begin{array}{l}\mathrm{miR}-146 \mathrm{a} \\
\mathrm{KO}\end{array}$ & 1.02 & 0.74 & 0.71 \\
\hline IL-6 & & & & MMP-9 & & & \\
\hline WT & 1.02 & 0.82 & 0.79 & WT & 1.08 & 1.27 & 1.35 \\
\hline $\begin{array}{l}\mathrm{miR}-146 a \\
\mathrm{KO}\end{array}$ & 0.80 & $0.72^{\star}$ & 0.74 & $\begin{array}{l}\mathrm{miR}-146 \mathrm{a} \\
\mathrm{KO}\end{array}$ & 1.03 & 0.73 & 1.39 \\
\hline IL-10 & & & & VEGF & & & \\
\hline WT & 0.82 & 0.70 & $0.66^{\star}$ & WT & 0.79 & 0.72 & $0.64^{*}$ \\
\hline $\begin{array}{l}\mathrm{miR}-146 \mathrm{a} \\
\mathrm{KO}\end{array}$ & $0.72^{\star}$ & $0.63^{\star}$ & $0.62^{\star}$ & $\begin{array}{l}\mathrm{miR}-146 \mathrm{a} \\
\mathrm{KO}\end{array}$ & $0.74^{\star}$ & $0.57^{\star \star}$ & $0.64^{\star}$ \\
\hline
\end{tabular}

Results are presented relative to the control levels of the particular strain. The expression level of these cytokines and chemokines were not significantly different between WT mice and miR-146a KO mice at any of the examined time-points. ${ }^{*} p<0.05,{ }^{* *} p<0.01,{ }^{* *} p<0.001, n=8-4$ in each group.

4 wd: 4 weeks demyelination. $2 d r: 2$ days (acute) remyelination, 2wr: 2 weeks (full) remyelination.

In two recent studies, miR-219 recombinant retrovirus injection into the corpus callosum attenuated demyelination in the CPZ model (45), and injection of miR-146a mimics into the demyelinated corpus callosum promoted remyelination (26). Only one recent study examined miRNA expression during CPZ-induced demyelination by microarray, but miRNAs were only evaluated in sorted CNPase-EGFP ${ }^{+}$cells with an OPC phenotype; only part of the differentially expressed miRNAs were presented, and those did not show miR-181b, miR-146a, or miR-193a (46).

We chose to further investigate the role of miR-146a in experimental demyelination, because (i) the expression levels of this miRNA was continuously increasing during de- and remyelination, which is in line with the finding of others (27); (ii) previous data indicated differential expression in different tissues and compartments of patients with MS, i.e., in brain lesions, body fluids, and cells (7, 8, 13, 18, 19); and (iii) miR$146 \mathrm{a}$ is known to be involved in regulation of the inflammatory response and survival processes of cells, including OPCs, which are relevant to MS demyelination (20-26, 32, 33).

miR-146a is highly expressed in microglia in the brain $(47,48)$. However, the observed increase in miR-146a in response to CPZ exposure in the corpus callosum cannot be solely explained by an increase in infiltrating microglia and macrophages, because the number of infiltrating microglia cells declines already 1 week after CPZ suspension $(49,50)$, and we found the highest level of miRNA-146a in the full remyelination phase, i.e., 2 weeks after suspending CPZ. Despite the increasing levels of miR-146a expression in the CNS, CPZ did not induce increased expression in a number of other organs, including the liver and the thymus, organs also affected by CPZ. This may indicate that the increased expression of miR-146a is unique to the CSN in response to CPZinduced de- and remyelination. Therefore, we also examined the level of miR-146a in response to physiological myelination in mice at early postnatal days, but we did not observe an increase in the expression level. These data suggest that the observed increase in miR-146a levels in the corpus callosum in response to $\mathrm{CPZ}$ exposure is a regulated process related to pathological demyelination and remyelination.

In order to further investigate the role of miR-146a during experimental de- and remyelination, we used a miR-146a KO strain (33). We compared the systemic and CNS effects of CPZ exposure between $\mathrm{KO}$ and WT mice. Mice are known to lose weight in response to $\mathrm{CPZ}$ exposure, and we have recently recognized thymus atrophy with loss of double-positive thymocytes as an additional systemic effect of CPZ exposure (39). Here, we found that $\mathrm{CPZ}$ also induced atrophy of another immune organ, the spleen. Previous data suggested that administration of $\mathrm{CPZ}$ ameliorates EAE and delays the progressive course of Theiler's murine encephalomyelitis $(51,52)$. It is possible that the additive effect of $\mathrm{CPZ}$ on primary and secondary immune organs may contribute to a deficiency of immune responses. We also found that miR-146a KO mice were protected against these systemic effects of CPZ: the atrophy of the thymus, spleen, and loss of body weight were all reduced in the $\mathrm{KO}$ mice. The most significant loss of weight was observed after 2 weeks of $\mathrm{CPZ}$ administration, and the protection was significant after 3 weeks. These data suggest that miRNA-146a may be involved in regulation of toxic responses and mitochondrial dysfunction, considering the mitochondrial effect of CPZ. Indeed, recent data indicate that differential expression and single nucleotide polymorphism of miR-146a can be related to drug-induced hepato- and cardiotoxicity $(53,54)$. In addition, miR-146a is one of the mitochondria-enriched miRNAs with potential targets on mitochondrial mRNAs, and it is most upregulated in senescent cells with mitochondrial dysfunction, altered fission, and fusion $(55,56)$.

Absence of miR-146a also reduced demyelination and axonal loss. The observed decrease in lesion size in miR-146a $\mathrm{KO}$ mice was accompanied by lower numbers of $\mathrm{Mac}^{+}$and $\mathrm{Iba}^{+}$macrophages/microglia, and a higher number of $\mathrm{CNP}^{+}$ myelinating oligodendrocytes in the corpus callosum during demyelination. Protective effects of miR-146a deficiency have been also shown in other degenerative experimental models. In a rat model for temporal lobe epilepsy (57) and in a mouse model for Alzheimer's disease (58), miR-146a was found to be upregulated in the brain, and inhibition by antagomiR-146a in these models led to decreased episodes of seizures and partly restored memory function, respectively. A very recent paper suggested that administration of miR-146a mimics into the corpus callosum of mice during remyelination enhanced remyelination and promoted OPC differentiation (27). In our study, miR-146 deficiency had no effect on remyelination and did not influence the number of OPCs during remyelination. 
Nevertheless, it reduced demyelination and axonal loss along with increased number of oligodendrocytes during demyelination. Since we did not find an increase of $\mathrm{NG}^{+}$OPCs in the $\mathrm{KO}$ mice during demyelination, the higher number of myelinating oligodendrocytes in the demyelinating corpus callosum may indicate increased survival of oligodendrocytes. This may also be related to change in the oligodendrocyte environment and in function of other resident cells due to the general absence of miR-146a. Interestingly, we observed a trend of reduced number of oligodendrocyte precursors in the $\mathrm{KO}$ mice during remyelination that may suggest that miR-146a may be beneficial during remyelination as previously suggested (27). These data may indicate a complex role of miR-146a in de- and remyelination, when examined at tissue level. The reduced axonal damage may be related to less demyelination, but axonal damage may be partly independent of demyelination in the CPZ model (59). Since mitochondrial alterations are important in axonal damage in MS, some of the protective effect in the KO mice may be related to the mitochondrial miR-146a pathways in the axons.

Next, we searched for proteins of validated target genes in our proteome database obtained during CPZ-induced de- and remyelination. We found two proteins to be downregulated during demyelination: SMAD4 and SNAP25. SMAD4 is involved in OPC migration and differentiation $(42,43)$, and SNAP25 is important in signal transduction of neurons and in neurotransmitter release (44). We, therefore, examined the levels of these two proteins in lysates of the corpus callosum dissected from the WT and miR-146a KO mice during de- and remyelination. The concentration of SNAP25 was increased in the miR-146a KO mice, as expected. SNAP25 was downregulated during demyelination in the miR-146a KO mice, but was not different from those in the WT mice during demyelination. Thus, ELISA results did not suggest differential regulation of SMAD4 and SNAP25 in the $\mathrm{KO}$ mice during demyelination.

miR-146a is a well-known negative regulator of the immune system which has been thoroughly investigated both in vivo and in vitro (21). We found that CCl2 was upregulated in WT mice in response to $\mathrm{CPZ}$ treatment, which is in line with results obtained by others (60). However, miR-146a KO mice expressed CCL2 in significantly lower levels during demyelination. CCL2 is a chemokine that is highly expressed by astrocytes in response to inflammatory events leading to attraction of immune cells, especially monocytes, to the inflammatory site (61). In the EAE model, conditional knockdown of CCL2 in astrocytes reduced the clinical score and infiltration by inflammatory microglia and macrophages, and delayed axonal damage in the spinal cord (62). This finding is in line with our observation that $\mathrm{Mac}^{+}$ cells were reduced in the miR-146a KO mice during demyelination along with decreased levels of CCL2. In addition, we found that TNF-RI and TNF-RII levels were increased in the WT mice, but not in the miR-146a KO mice, and that there was a significant difference in the expression levels of these proteins between WT mice and miR-146a KO mice during demyelination. Previous data showed that TNF facilitated the toxic effect of $\mathrm{CPZ}$ on oligodendrocytes in vitro, and induced the depletion of microglia in vivo, the main source of cytokine and chemokine expression in the brain. This in turn resulted in protection against CPZ-induced demyelination (63). Therefore, it is likely that the reduction of TNF-RI, TNF-RII, and CCL2 in the miR-146a KO mice contributed to the protection against $\mathrm{CPZ}$-induced demyelination. In this study, we did not examine the cellular source of these dysregulated molecules in the KO mice. The reduced levels of TNF-RI, TNF-RII, and CCL2 may reflect differential expression by different cells in the demyelinating corpus callosum, and this can influence the ultimate protective versus detrimental effect; the observed protection from CPZ-induced demyelination is a combinatory effect of miR-146a deficiency in all cell types, and we cannot exclude the possibility that lack of miR-146a in particular cell types could be harmful. We hypothesize that pro-apoptotic properties of miR-146a $(23,24,32,64)$ may also contribute to decreased demyelination, increased number of myelinating oligodendrocytes and reduced axonal loss during the demyelination phase induced by $\mathrm{CPZ}$ in $\mathrm{KO}$ mice.

In summary, here we used a comprehensive and unbiased approach to identify three miRNAs, miR-146a, miR-181b, and miR-193a, which were differentially regulated in the corpus callosum in response to $\mathrm{CPZ}$ exposure. We further investigated the effect of absence of miR-146a, and found that the number of oligodendrocytes was higher during demyelination in miR146a KO mice, and demyelination and axonal loss were reduced. In addition, there was no increase of CCL2 in the demyelinating corpus callosum of the $\mathrm{KO}$ in contrast to the WT mice, and CCL2 levels were lower in the KO mice; this may explain the observed fewer number of infiltrating macrophages/microglia. Contrary to WT mice, there was no increase in the levels of TNF receptors in the corpus callosum of the $\mathrm{KO}$ mice in response to $\mathrm{CPZ}$, indicating reduced inflammatory changes that may be related to the reduced number of macrophages/microglia. Altogether, these findings may suggest increased survival of oligodendrocytes, reduced production of CCL2 by astrocytes, less microglia/macrophage activation and TNF receptor expression in the KO mice, which result in reduced demyelination and axonal loss. We also observed a mild increase in $\mathrm{NG}^{+}$ OPCs during remyelination in the $\mathrm{KO}$ mice that may support previous data indicating the beneficial role of miR-146a during remyelination (33). Additional studies should address the cellular source of the altered molecules in the corpus callosum, and if administration of antagomirs in WT mice results in similar changes.

\section{ETHICS STATEMENT}

All animal experiments complied with the EU Directive 2010/63/EU for animal experiments. Protocols and guidelines approved by the Danish Animal Health Care Committee (201415-00369).

\section{AUTHOR CONTRIBUTIONS}

NM performed CPZ experiments, quantitative PCR, Meso Scale arrays, pathway analysis, and wrote the manuscript. VM performed microarray experiments and contributed to writing 
the manuscript. GS contributed to CPZ experiments and proteomics. ME, JO, AW, and ET contributed to CPZ and related experiments. AN performed proteomics and contributed to writing the manuscript. MP contributed to microdissection. FG contributed to supervising part of the CPZ experiments, proteomics, and contributed to writing the manuscript. ML supervised and designed proteomics. HL performed histology and quantification of de- and remyelination and cells. EB participated in design of the study, supervision, and contributed to writing the manuscript. TO participated in design of the study, supervision, and contributed to writing the manuscript. AS participated in design of the study, supervision, and contributed to writing the manuscript. ZI conceived the idea, designed the study, supervised, and contributed to writing the manuscript.

\section{REFERENCES}

1. Lassmann H. Multiple sclerosis: lessons from molecular neuropathology. Exp Neurol (2014) 262:2-7. doi:10.1016/j.expneurol.2013.12.003

2. Larochelle C, Uphaus T, Prat A, Zipp F. Secondary progression in multiple sclerosis: neuronal exhaustion or distinct pathology? Trends Neurosci (2016) 39:325-39. doi:10.1016/j.tins.2016.02.001

3. Friedman RC, Farh KKH, Burge CB, Bartel DP. Most mammalian mRNAs are conserved targets of microRNAs. Genome Res (2009) 19:92-105. doi:10.1101/ gr.082701.108

4. van Rooij E, Kauppinen S. Development of microRNA therapeutics is coming of age. EMBO Mol Med (2014) 6:851-64. doi:10.15252/emmm.201100899

5. Bouchie A. First microRNA mimic enters clinic. Nat Biotechnol (2013) 31:577. doi:10.1038/nbt0713-577

6. Janssen HL, Reesink HW, Lawitz EJ, Zeuzem S, Rodriguez-Torres M, Patel K, et al. Treatment of HCV infection by targeting microRNA. N Engl J Med (2013) 368:1685-94. doi:10.1056/NEJMoa1209026

7. Martin NA, Illes Z. Differentially expressed microRNA in multiple sclerosis: a window into pathogenesis? Clin Exp Neuroimmunol (2014) 5:149-61. doi:10.1111/cen3.12131

8. Junker A, Krumbholz M, Eisele S, Mohan H, Augstein F, Bittner R, et al. MicroRNA profiling of multiple sclerosis lesions identifies modulators of the regulatory protein CD47. Brain (2009) 132:3342-52. doi:10.1093/brain/ awp300

9. Dutta R, Chomyk AM, Chang A, Ribaudo MV, Deckard SA, Doud MK, et al. Hippocampal demyelination and memory dysfunction are associated with increased levels of the neuronal microRNA miR-124 and reduced AMPA receptors. Ann Neurol (2013) 73:637-45. doi:10.1002/ana.23860

10. Cox MB, Cairns MJ, Gandhi KS, Carroll AP, Moscovis S, Stewart GJ, et al. MicroRNAs miR-17 and miR-20a inhibit T cell activation genes and are under-expressed in MS whole blood. PLoS One (2010) 5(8):e12132. doi:10.1371/journal.pone.0012132

11. Keller A, Leidinger P, Steinmeyer F, Stähler C, Franke A, HemmrichStanisak G, et al. Comprehensive analysis of microRNA profiles in multiple sclerosis including next-generation sequencing. Mult Scler (2014) 20:295-303. doi:10.1177/1352458513496343

12. Søndergaard HB, Hesse D, Krakauer M, Sørensen PS, Sellebjerg F. Differential microRNA expression in blood in multiple sclerosis. Mult Scler (2013) 19:1849-57. doi:10.1177/1352458513490542

13. Fenoglio C, Cantoni C, De Riz M, Ridolfi E, Cortini F, Serpente M, et al. Expression and genetic analysis of miRNAs involved in CD4+ cell activation in patients with multiple sclerosis. Neurosci Lett (2011) 504:9-12. doi:10.1016/j.neulet.2011.08.021

14. Gandhi R, Healy B, Gholipour T, Egorova S, Musallam A, Hussain MS, et al. Circulating microRNAs as biomarkers for disease staging in multiple sclerosis. Ann Neurol (2013) 73:729-40. doi:10.1002/ana.23880

15. Zhang J, Cheng Y, Cui W, Li M, Li B, Guo L. MicroRNA-155 modulates Th1 and Th17 cell differentiation and is associated with multiple sclerosis

\section{ACKNOWLEDGMENTS}

We thank Ulla Damgaard Munk and Helle Vinsløv Jensen for expert technical assistance. We thank Nikolett Hanák, Viktória Dellaszéga-Lábas, and Éva Rebeka Szabó, PhD (Semmelweis University) for their technical assistance.

\section{FUNDING}

Lundbeckfonden R118-A11472, OTKA-NN109841, OTKAK77892, Region of Southern Denmark 14/24200, Jascha Fonden 5589, Direktør Ejnar Jonasson kaldet Johnsen og hustrus mindelegat 5609, Odense University Hospital (OUH) A474, Fonden for Laegevidenskabensfremme 13-267, GINOP 2.3.2-15-201600049, GINOP 2.3.3-15-2016-00025.

and experimental autoimmune encephalomyelitis. J Neuroimmunol (2014) 266:56-63. doi:10.1016/j.jneuroim.2013.09.019

16. Haghikia A, Haghikia A, Hellwig K, Baraniskin A, Holzmann A, Décard BF, et al. Regulated microRNAs in the CSF of patients with multiple sclerosis: a case-control study. Neurology (2012) 79:2166-70. doi:10.1212/WNL. 0b013e3182759621

17. Bergman P, Piket E, Khademi M, James T, Brundin L, Olsson T, et al. Circulating miR-150 in CSF is a novel candidate biomarker for multiple sclerosis. Neurol Neuroimmunol Neuroinflamm (2016) 3(3):e219. doi:10.1212/NXI. 0000000000000219

18. Yang D, Wang W-Z, Zhang X-M, Yue H, Li B, Lin L, et al. MicroRNA expression aberration in Chinese patients with relapsing remitting multiple sclerosis. J Mol Neurosci (2014) 52:131-7. doi:10.1007/s12031-013-0138-x

19. Waschbisch A, Atiya M, Linker RA, Potapov S, Schwab S, Derfuss T. Glatiramer acetate treatment normalizes deregulated microRNA expression in relapsing remitting multiple sclerosis. PLoS One (2011) 6(9):e24604. doi:10.1371/ journal.pone.0024604

20. Chan EKL, Ceribelli A, Satoh M. MicroRNA-146a in autoimmunity and innate immune responses. Ann Rheum Dis (2013) 72(Suppl 2):ii90-5. doi:10.1136/ annrheumdis-2012-202203

21. Saba R, Sorensen DL, Booth SA. MicroRNA-146a: a dominant, negative regulator of the innate immune response. Front Immunol (2014) 5:578. doi:10.3389/ fimmu.2014.00578

22. Taganov KD, Boldin MP, Chang K-J, Baltimore D. NF-kappaB-dependent induction of microRNA miR-146, an inhibitor targeted to signaling proteins of innate immune responses. Proc Natl Acad Sci U S A (2006) 103:12481-6. doi:10.1073/pnas.0605298103

23. Mei J, Bachoo R, Zhang C-L. MicroRNA-146a inhibits glioma development by targeting Notch1. Mol Cell Biol (2011) 31:3584-92. doi:10.1128/MCB.05821-11

24. Zhang B, Wang LL, Ren RJ, Dammer EB, Zhang YF, Huang Y, et al. MicroRNA146a represses LRP2 translation and leads to cell apoptosis in Alzheimer's disease. FEBS Lett (2016) 590:2190-200. doi:10.1002/1873-3468.12229

25. Santra M, Zhang ZG, Yang J, Santra S, Santra S, Chopp M, et al. Thymosin $\beta 4$ up-regulation of microRNA-146a promotes oligodendrocyte differentiation and suppression of the toll-like proinflammatory pathway. J Biol Chem (2014) 289:19508-18. doi:10.1074/jbc.M113.529966

26. Liu XS, Chopp M, Pan WL, Wang XL, Fan BY, Zhang Y, et al. MicroRNA146a promotes oligodendrogenesis in stroke. Mol Neurobiol (2017) 54: 227-37. doi:10.1007/s12035-015-9655-7

27. Zhang J, Zhang ZG, Lu M, Wang X, Shang X, Elias SB, et al. miR-146a promotes remyelination in a cuprizone model of demyelinating injury. Neuroscience (2017) 348:252-63. doi:10.1016/j.neuroscience.2017.02.029

28. Kipp M, Nyamoya S, Hochstrasser T, Amor S. Multiple sclerosis animal models: a clinical and histopathological perspective. Brain Pathol (2017) 27:123-37. doi:10.1111/bpa.12454

29. Illes Z, Lassmann H, Gallyas F Jr. A novel concept of treatment in MS: targeting both oligodendrocyte death and inflammatory processes by inhibiting poly(adp-ribose) polymerase. In: Gran B, Yamamura T, editors. Multiple 
Sclerosis Immunology - A Foundation for Current and Future Treatments. New York: Springer (2013). p. 315-40.

30. Praet J, Guglielmetti C, Berneman Z, Van der Linden A, Ponsaerts P. Cellular and molecular neuropathology of the cuprizone mouse model: clinical relevance for multiple sclerosis. Neurosci Biobehav Rev (2014) 47:485-505. doi:10.1016/j.neubiorev.2014.10.004

31. Hiremath MM, Chen VS, Suzuki K, Ting JP, Matsushima GK. MHC class II exacerbates demyelination in vivo independently of T cells. J Neuroimmunol (2008) 203:23-32. doi:10.1016/j.jneuroim.2008.06.034

32. Zhou X, Su S, Li S, Pang X, Chen C, Li J, et al. MicroRNA-146a downregulation correlates with neuroprotection and targets pro-apoptotic genes in cerebral ischemic injury in vitro. Brain Res (2016) 1648:136-43. doi:10.1016/j. brainres.2016.07.034

33. Boldin MP, Taganov KD, Rao DS, Yang L, Zhao JL, Kalwani M, et al. miR-146a is a significant brake on autoimmunity, myeloproliferation, and cancer in mice. J Exp Med (2011) 208:1189-201. doi:10.1084/jem. 20101823

34. Sidman RL. Atlas of the Mouse Brain and Spinal Cord. Cambridge: Harvard University Press (1971).

35. Marik C, Felts PA, Bauer J, Lassmann H, Smith KJ. Lesion genesis in a subset of patients with multiple sclerosis: a role for innate immunity? Brain (2007) 130:2800-15. doi:10.1093/brain/awm236

36. Palmisano G, Parker BL, Engholm-Keller K, Lendal SE, Kulej K, Schulz M, et al. A novel method for the simultaneous enrichment, identification, and quantification of phosphopeptides and sialylated glycopeptides applied to a temporal profile of mouse brain development. Mol Cell Proteomics (2012) 11:1191-202. doi:10.1074/mcp.M112.017509

37. Engholm-Keller K, Larsen MR. Improving the phosphoproteome coverage for limited sample amounts using $\mathrm{TiO}_{2}$-SIMAC-HILIC (TiSH) phosphopeptide enrichment and fractionation. Methods Mol Biol (2016) 1355:161-77. doi:10.1007/978-1-4939-3049-4_11

38. Semple BD, Blomgren K, Gimlin K, Ferriero DM, Noble-Haeusslein LJ. Brain development in rodents and humans: identifying benchmarks of maturation and vulnerability to injury across species. Prog Neurobiol (2013) 107:1-16. doi:10.1016/j.pneurobio.2013.04.001

39. Solti I, Kvell K, Talaber G, Veto S, Acs P, Gallyas F Jr, et al. Thymic atrophy and apoptosis of CD4+ CD8+ thymocytes in the cuprizone model of multiple sclerosis. PLoS One (2015) 10(6):e0129217. doi:10.1371/journal.pone. 0129217

40. Chou C-H, Chang N-W, Shrestha S, Hsu SD, Lin YL, Lee WH, et al. miRTarBase 2016: updates to the experimentally validated miRNA-target interactions database. Nucleic Acids Res (2016) 44:239-47. doi:10.1093/nar/ gkv1258

41. Mi H, Huang X, Muruganujan A, Tang H, Mills C, Kang D, et al. PANTHER version 11: expanded annotation data from gene ontology and reactome pathways, and data analysis tool enhancements. Nucleic Acids Res (2017) 45:183-9. doi:10.1093/nar/gkw1138

42. Choe Y, Huynh T, Pleasure SJ. Migration of oligodendrocyte progenitor cells is controlled by transforming growth factor $\beta$ family proteins during corticogenesis. J Neurosci (2014) 34:14973-83. doi:10.1523/JNEUROSCI. 1156-14.2014

43. Pedraza CE, Taylor C, Pereira A, Seng M, Tham CS, Izrael M, et al. Induction of oligodendrocyte differentiation and in vitro myelination by inhibition of rho-associated kinase. ASN Neuro (2014) 6:4. doi:10.1177/ 1759091414538134

44. Rizo J, Südhof TC. The membrane fusion enigma: SNAREs, Sec1/Munc18 proteins, and their accomplices - guilty as charged? Annu Rev Cell Dev Biol (2012) 28:279-308. doi:10.1146/annurev-cellbio-101011-155818

45. Liu S, Ren C, Qu X, Wu X, Dong F, Chand YK, et al. miR-219 attenuates demyelination in cuprizone-induced demyelinated mice by regulating monocarboxylate transporter 1. Eur J Neurosci (2017) 45:249-59. doi:10.1111/ ejn. 13485

46. Kuypers NJ, Bankston AN, Howard RM, Beare JE, Whittemore SR. Remyelinating oligodendrocyte precursor cell miRNAs from the Sfmbt2 cluster promote cell cycle arrest and differentiation. J Neurosci (2016) 36:1698-710. doi:10.1523/JNEUROSCI.1240-15.2016

47. Jovičić A, Roshan R, Moisoi N, Pradervand S, Moser R, Pillai B, et al. Comprehensive expression analyses of neural cell-type-specific miRNAs identify new determinants of the specification and maintenance of neuronal phenotypes. Ann Intern Med (2013) 158:5127-37. doi:10.1523/JNEUROSCI. 0600-12.2013

48. Wang W-X, Visavadiya NP, Pandya JD, Nelson PT, Sullivan PG, Springer JE. Mitochondria-associated microRNAs in rat hippocampus following traumatic brain injury. Exp Neurol (2015) 265:84-93. doi:10.1016/j.expneurol. 2014.12.018

49. Remington LT, Babcock AA, Zehntner SP, Owens T. Microglial recruitment, activation, and proliferation in response to primary demyelination. Am J Pathol (2007) 170:1713-24. doi:10.2353/ajpath.2007.060783

50. Gudi V, Gingele S, Skripuletz T, Stangel M. Glial response during cuprizoneinduced de- and remyelination in the CNS: lessons learned. Front Cell Neurosci (2014) 8:73. doi:10.3389/fncel.2014.00073

51. Maña P, Fordham SA, Staykova MA, Correcha M, Silva D, Willenborg DO, et al. Demyelination caused by the copper chelator cuprizone halts $\mathrm{T}$ cell mediated autoimmune neuroinflammation. J Neuroimmunol (2009) 210:13-21. doi:10.1016/j.jneuroim.2009.02.013

52. Herder V, Hansmann F, Stangel M, Schaudien D, Rohn K, Baumgärtner W, et al. Cuprizone inhibits demyelinating leukomyelitis by reducing immune responses without virus exacerbation in an infectious model of multiple sclerosis. J Neuroimmunol (2012) 244:84-93. doi:10.1016/j.jneuroim.2012. 01.010

53. Holmgren G, Synnergren J, Andersson CX, Lindahl A, Sartipy P. MicroRNAs as potential biomarkers for doxorubicin-induced cardiotoxicity. Toxicol In Vitro (2016) 34:26-34. doi:10.1016/j.tiv.2016.03.009

54. Fang C, Li XP, Gong WJ, Wu NY, Tang J, Yin JY, et al. Age-related common miRNA polymorphism associated with severe toxicity in lung cancer patients treated with platinum-based chemotherapy. Clin Exp Pharmacol Physiol (2017) 44(Suppl 1):21-9. doi:10.1111/1440-1681.12704

55. Dasgupta N, Peng Y, Tan Z, Ciraolo G, Wang D, Li R. miRNAs in mtDNA-less cell mitochondria. Cell Death Discov (2015) 1:15004. doi:10.1038/ cddiscovery.2015.4

56. Rippo MR, Olivieri F, Monsurrò V, Prattichizzo F, Albertini MC, Procopio AD. MitomiRs in human inflamm-aging: a hypothesis involving miR-181a, miR-34a and miR-146a. Exp Gerontol (2014) 56:154-63. doi:10.1016/j.exger. 2014.03.002

57. He F, Liu B, Meng Q, Sun Y, Wang W, Wang C. Modulation of miR-146a/ complement factor $\mathrm{H}$-mediated inflammatory responses in a rat model of temporal lobe epilepsy. Biosci Rep (2016) 36:e0433. doi:10.1042/ BSR20160290

58. Wang G, Huang Y, Wang L-L, Zhang YF, Xu J, Zhou Y, et al. MicroRNA-146a suppresses ROCK1 allowing hyperphosphorylation of tau in Alzheimer's disease. Sci Rep (2016) 6:26697. doi:10.1038/srep26697

59. Yoshikawa K, Palumbo S, Toscano CD, Bosetti F. Inhibition of 5lipoxygenase activity in mice during cuprizone-induced demyelination attenuates neuroinflammation, motor dysfunction and axonal damage. Prostaglandins Leukot Essent Fatty Acids (2011) 85:43-52. doi:10.1016/j. plefa.2011.04.022

60. Skripuletz T, Hackstette D, Bauer K, Gudi V, Pul R, Voss E, et al. Astrocytes regulate myelin clearance through recruitment of microglia during cuprizone-induced demyelination. Brain (2013) 136:147-67. doi:10.1093/ brain/aws 262

61. Semple BD, Kossmann T, Morganti-Kossmann MC. Role of chemokines in CNS health and pathology: a focus on the CCL2/CCR2 and CXCL8/ CXCR2 networks. J Cereb Blood Flow Metab (2010) 30:459-73. doi:10.1038/ jcbfm.2009.240

62. Moreno M, Bannerman P, Ma J, Guo F, Miers L, Soulika AM, et al. Conditional ablation of astroglial CCL2 suppresses CNS accumulation of M1 macrophages and preserves axons in mice with MOG peptide EAE. J Neurosci (2014) 34:8175-85. doi:10.1523/JNEUROSCI.1137-14.2014

63. Pasquini LA, Calatayud CA, Bertone Uña AL, Millet V, Pasquini JM, Soto EF. The neurotoxic effect of cuprizone on oligodendrocytes depends on the presence of pro-inflammatory cytokines secreted by microglia. Neurochem Res (2007) 32:279-92. doi:10.1007/s11064-006-9165-0

64. Liu R, Li W, Wu C. A functional polymorphism in the pre-miR-146a gene influences the prognosis of glioblastoma multiforme by interfering with the balance between Notch1 and Notch2. Mol Med Rep (2015) 12:5475-81. doi:10.3892/mmr.2015.4067 
Conflict of Interest Statement: The authors declare that the research was conducted in the absence of any commercial or financial relationships that could be construed as a potential conflict of interest.

Copyright (C) 2018 Martin, Molnar, Szilagyi, Elkjaer, Nawrocki, Okarmus, Wlodarczyk, Thygesen, Palkovits, Gallyas, Larsen, Lassmann, Benedikz, Owens,
Svenningsen and Illes. This is an open-access article distributed under the terms of the Creative Commons Attribution License (CC BY). The use, distribution or reproduction in other forums is permitted, provided the original author(s) and the copyright owner are credited and that the original publication in this journal is cited, in accordance with accepted academic practice. No use, distribution or reproduction is permitted which does not comply with these terms. 